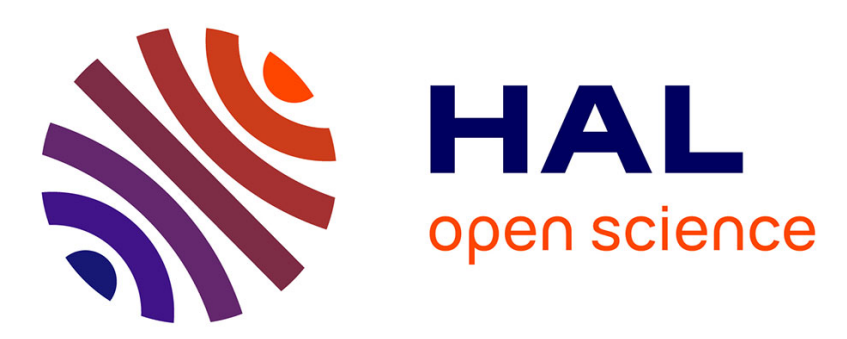

\title{
Remote vibration measurement: A wireless passive surface acoustic wave resonator fast probing strategy
}

\author{
J.-M. Friedt, C. Droit, S. Ballandras, S. Alzuaga, G. Martin, P. Sandoz
}

\section{To cite this version:}

J.-M. Friedt, C. Droit, S. Ballandras, S. Alzuaga, G. Martin, et al.. Remote vibration measurement: A wireless passive surface acoustic wave resonator fast probing strategy. Review of Scientific Instruments, 2012, 83 (5), pp.055001. 10.1063/1.4705728. hal-00762519

\section{HAL Id: hal-00762519 \\ https://hal.science/hal-00762519}

Submitted on 17 May 2021

HAL is a multi-disciplinary open access archive for the deposit and dissemination of scientific research documents, whether they are published or not. The documents may come from teaching and research institutions in France or abroad, or from public or private research centers.
L'archive ouverte pluridisciplinaire HAL, est destinée au dépôt et à la diffusion de documents scientifiques de niveau recherche, publiés ou non, émanant des établissements d'enseignement et de recherche français ou étrangers, des laboratoires publics ou privés. 


\title{
Remote vibration measurement: A wireless passive surface acoustic wave resonator fast probing strategy
}

\author{
J.-M. Friedt, ${ }^{1, \text { a) }}$ C. Droit, ${ }^{1}$ S. Ballandras, ${ }^{2, b)}$ S. Alzuaga, ${ }^{2}$ G. Martin, ${ }^{2}$ and P. Sandoz ${ }^{3}$ \\ ${ }^{1}$ SENSeOR SAS, Besançon, France \\ ${ }^{2}$ FEMTO-ST, Time and Frequency Department, UMR CNRS 6174, University of Franche-Comté, \\ Besançon, France \\ ${ }^{3}$ FEMTO-ST, Applied Mechanics Department, UMR CNRS 6174, University of Franche-Comté, \\ Besançon, France
}

(Received 1 February 2012; accepted 30 March 2012; published online 1 May 2012)

\begin{abstract}
Surface acoustic wave (SAW) resonators can advantageously operate as passive sensors which can be interrogated through a wireless link. Amongst the practical applications of such devices, structural health monitoring through stress measurement and more generally vibration characteristics of mechanical structures benefit from the ability to bury such sensors within the considered structure (wireless and battery-less). However, measurement bandwidth becomes a significant challenge when measuring wideband vibration characteristics of mechanical structures. A fast SAW resonator measurement scheme is demonstrated here. The measurement bandwidth is limited by the physical settling time of the resonator $(Q / \pi$ periods), requiring only two probe pulses through a monostatic RADAR-like electronic setup to identify the sensor resonance frequency and hence stress on a resonator acting as a strain gauge. A measurement update rate of $4800 \mathrm{~Hz}$ using a high quality factor SAW resonator operating in the $434 \mathrm{MHz}$ Industrial, Scientific and Medical band is experimentally demonstrated. (C) 2012 American Institute of Physics. [http://dx.doi.org/10.1063/1.4705728]
\end{abstract}

\section{INTRODUCTION}

Piezoelectric transducers acting as passive sensors probed through a wireless link have demonstrated extended robustness and interrogation range ${ }^{1,2}$ compared to silicon based radiofrequency identification devices. ${ }^{3}$ Because such devices act linearly in the conversion of the incoming electromagnetic wave to the acoustic propagating wave, the interrogation range is not limited by the output power reaching a rectifier diode threshold voltage but solely by the receiver noise level and the ability to identify the sensor signal above this noise.

Surface acoustic wave (SAW) sensors are designed along two main approaches:

1. Wideband delay lines in which a short pulse launched by an interdigitated transducer (IDT) propagates over the free piezoelectric substrate surface with a velocity dependent on the environmental physical property and hence a time delay representative of this physical property.

2. Narrowband resonators in which an acoustic wave is excited by the above-mentioned IDT placed between two Bragg mirrors, exhibiting a resonant frequency dependent on the physical property under consideration.

An acoustic delay line being a wideband device, it should in principle be the class of sensor fastest to probe, with typical time delay in the $5 \mu$ s range, yielding $200 \mathrm{kHz}$ measurement rate using high speed analog-to-digital converters and fast storage media. Such hardware is however power consuming and hardly compatible with embedded applications.

a)Electronic mail: jmfriedt@femto-st.fr; http://jmfriedt.free.fr.

b) Also at SENSeOR SAS, Besançon, France.
Classical measurement techniques-frequency sweep probing of the returned power of either delay line or resonator, or impulse response measurement of the time domain response of both types of transducer-often exhibit limited bandwidth of the measured physical quantity. This is due to the large number of sampled frequencies and to the settling time of the tunable frequency source/sampling time of the digitization of the returned signal at each frequency. ${ }^{4}$ While hardly an issue for temperature measurements whose bandwidth is limited by the thermal inertia of the sensor to a few hertz, the measurement refresh rate becomes a significant parameter when probing strain gauges acting as sensors on vibrating elements.

In this paper, after identifying some of the physical limitations of the acoustic resonator probing and proposing a measurement algorithm requiring only two measurement frequencies, the measurement of the stress on a music tuning fork fitted with a surface acoustic wave (SAW) resonator strain gauge is demonstrated. A sampling rate of $4800 \mathrm{~Hz}$ is reached using a high $Q(Q=13300)$ SAW resonator operating at $434 \mathrm{MHz}$, i.e., half the theoretically estimated maximum measurement rate.

\section{FAST MEASUREMENT ALGORITHM}

\section{A. Algorithm principles and interest}

An algorithm dedicated to radiofrequency SAW resonator probing has been described in a previous work, ${ }^{5}$ based on a frequency-modulation (FM) to amplitude-modulation (AM) conversion. ${ }^{6}$ The emphasis in presenting this algorithm was on its ability to improve the measured frequency resolution by exploiting the linear phase vs. frequency 
relationship, thus improving the feedback algorithm on the identified resonant frequency. However, a significant hindrance of the full FM probing implementation of the resonator response is the long interrogation duration since multiple FM periods are needed for phase extraction, either using an analog low pass filter after a mixer stage or a digital low pass filter acting on the recorded AM samples. ${ }^{5}$ On the other hand, a fully digital implementation of the FM-AM algorithm demonstrates that only two measurements are actually needed for extracting an information similar to the so-called phase information in the previous implementation, namely the maximum and minimum FM frequencies: the returned power difference at these two frequencies exhibits a behaviour reminiscent of the phase behaviour in an FM-AM conversion system. Hence, the phase feedback algorithm becomes a feedback control aimed at keeping the magnitude response balanced when probing the resonator with only two frequencies, one located below and one located above the resonance frequency.

The digital implementation ${ }^{7}$ of the interrogation unit probes the power returned by the SAW sensors at two frequencies located at $f-f_{\text {step }}$ and $f+f_{\text {step }}$ with $f_{\text {step }}$ the FM frequency excursion and $f$ an hypothetical central FM frequency. The latter is never practically generated but is assumed to be close to the resonance frequency $f_{0}$ of the sensor. The algorithm thus designed for tracking the resonance frequency controls the interrogation frequencies: $f-f_{\text {step }}$ and $f+f_{\text {step }}$, respectively, in order to keep the returned signals $y\left(f-f_{\text {step }}\right)$ and $y(f$ $+f_{\text {step }}$ ) equal (Fig. 1). Therefore, rather than probing the sensor response at a large number of frequencies and consequently suffering for a long interrogation process, this strategy minimizes the number of probe frequencies needed to the two extreme frequencies of the FM signal with excursion $f_{\text {step }}$. The measurement bandwidth is thus increased while keeping the measurement accuracy.

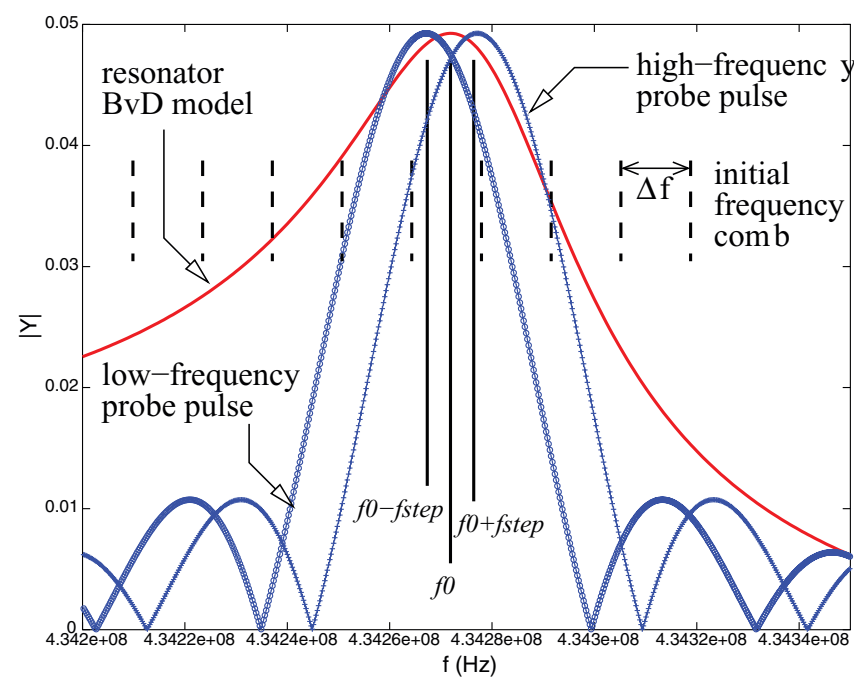

FIG. 1. Simulation of the two-point interrogation strategy-each pulse is generated as a rectangular $30 \mu$ s long window-probing a resonator fitted with a Butterworth-van Dyke model $\left(C_{0}=1.7 \mathrm{pF}, C_{1}=0.56 \mathrm{~F}\right.$, $L_{1}=241 \mu \mathrm{H}, R_{1}=73 \Omega$ ).

\section{B. Frequency step and proportional feedback gain identification}

Hence, considering two resonator measurements $y(f$ $\left.-f_{\text {step }}\right)$ and $y\left(f+f_{\text {step }}\right)$, the feedback loop aims at identifying the resonance frequency $f_{0}$ so that $y\left(f_{0}-f_{\text {step }}\right)=y\left(f_{0}\right.$ $\left.+f_{\text {step }}\right)$. The expression of the resonator response following the Butterworth-Van Dyke (BvD) equivalent circuit is not trivial and does not yield analytical feedback control loop coefficients. We use instead a second order polynomial fit of the resonator response to define the proportional feedback control loop gain factor. Indeed, during an initialization step of the algorithm, the SAW sensor response is probed along a frequency comb with spacing $\Delta f$ (Fig. 1) selected so that, under the assumption of the SAW resonator quality factor is known, at least three returned magnitude measurements fit within the bandpass of the resonator. These three measurements provide a unique parabola equation describing the resonator returned power law close to the resonance, whose shape is assumed throughout this document to be constant whatever the physical quantity under investigation acting on the SAW sensor (constant quality factor and varying resonance frequency assumptions). Thus, assuming three returned signal measurements $y_{i}(i \in[1, \ldots, 3])$ obtained at $f-\Delta f, f$, and $f+\Delta f$ respectively during the initialization process, with $y_{2}>y_{1}, y_{3}$, the resonator parabolic fit curvature then expresses as ${ }^{7}$

$$
A=\frac{y_{3}+y_{1}-2 y_{2}}{2(\Delta f)^{2}}
$$

and the resonance frequency $f_{0}$ so that the power law $y=A(f$ $\left.-f_{0}\right)^{2}+B$ fitting at best the measured return power is given by $f_{0}=f-\frac{y_{2}-y_{1}}{2 \times A \times \Delta f}$. The curvature $A$ will be used to estimate the gain of the feedback loop relating the output central probe frequency during the two-point approach to the returned power difference $\delta y=y\left(f+f_{\text {step }}\right)-y\left(f-f_{\text {step }}\right)$ (also known as the error signal, expected to vanish due to the feedback loop acting on $f$ and aimed at bringing $f$ close to the resonance frequency $f_{0}$ ).

Expressing the fact that at resonance, when $f=f_{0}, \delta y=0$ and using the locally parabolic approximation of the resonator shape, this expression becomes $f_{0}=f-\frac{y\left(f-f_{\text {step }}\right)-y\left(f+f_{\text {step }}\right)}{2 f_{\text {step }} \frac{y_{3}+y_{1}-2 y_{2}}{(\Delta f)^{2}}}$ : the correction to the current value of the probed frequency $f$ is

$$
\frac{(\Delta f)^{2}}{2 f_{\text {step }}} \times \frac{y\left(f-f_{\text {step }}\right)-y\left(f+f_{\text {step }}\right)}{y_{3}+y_{1}-2 y_{2}}
$$

and the slope of this proportional correction is

$$
\frac{(\Delta f)^{2}}{2 f_{\text {step }}} \times \frac{1}{y_{3}+y_{1}-2 y_{2}}=\frac{1}{4 A f_{\text {step }}} .
$$

The ability to control the feedback loop on the emitted probe frequency was thus demonstrated in order to track the resonance frequency using only two resonator measurements. The remaining limitation on the measurement speed is then the duration of the resonator probing. Since a resonator ${ }^{8}$ operating at $f_{0}$ and exhibiting a quality factor $Q$ is characterized by a time constant (for loading or unloading energy to $1 / e=63 \%$ of the asymptotic value) of $\tau=Q /\left(\pi f_{0}\right)$, the measurement duration is of the order of $2 \tau$. With $Q=13300$ at $f_{0}=434 \mathrm{MHz}$, this measurement duration is $20 \mu \mathrm{s}$ 
corresponding to a measurement rate of $50 \mathrm{kHz}$. Our practical implementation of the measurement algorithm waits during $5 \tau$ for loading the resonator to $99.3 \%$ of its asymptotic value, thus providing a narrowband interrogation pulse aimed at fully loading the resonator, and $5 \tau$ for unloading the resonator and making sure that each successive measurement is independent by starting from an unloaded resonator. Hence, the theoretical maximum update rate of our two-point interrogation strategy is $10 \mathrm{kHz}$.

One last free parameter is $f_{\text {step }}$, acting as the FM excursion in the previous formalism. ${ }^{5}$ The quantification of the returned power digitized for processing purpose constraints this parameter: under the worst condition when $y\left(f-f_{\text {step }}\right)=0$ and $y\left(f+f_{\text {step }}\right)=y_{\max }$ with $y_{\max }$ the maximum quanticized value (e.g., 4096 in the case of a 12 bit analog-to-digital converter), the feedback loop should move to $f$ by less than $f_{\text {step }}$. Using the previous expression of the slope relating the frequency correction to the measured amplitude error, a condition on $f_{\text {step }}$ is $y_{\text {max }} \times \frac{(\Delta f)^{2}}{2 f_{\text {step }}} \times \frac{1}{y_{3}+y_{1}-2 y_{2}}<f_{\text {step }}$ and under appropriate conditions of improved signal to noise ratio, $y_{3}+y_{1}-2 y_{2} \sim y_{\max }$ so that $(\Delta f)^{2}<2 f_{\text {step }}^{2}$. The FM excursion should be selected larger than the initialization frequency step, which has already been selected at one third of the width at half height $1 / 3$ $\times f_{0} / Q$ of the resonator. ${ }^{7}$ Selecting a large enough FM excursion reduces the chance of amplifying the amplitude measurement error and generating frequency corrections above $f_{\text {step }}$. However, such a condition means probing the resonator far from its resonance, with two consequences:

- Assumptions concerning the BvD polynomial fit are no longer valid since the returned power dependency departs significantly from a parabolic shape.

- Most significantly, the resonator is probed far from its resonance, and hence loads little energy (overlap in the frequency domain of the probe pulse spectrum and resonator transfer function). The consequence is a reduced interrogation distance since the returned power has to remain above the receiver noise level.

Despite the chance of saturating the feedback loop for large physical quantity variations yielding large resonance frequency variations, the use of $f_{\text {step }}=5 \mathrm{kHz}$ (smaller than $\Delta f=15 \mathrm{kHz}$ ) has been selected all along this work to preserve the interrogation distance by keeping the probe pulse frequencies close to the resonance frequency.

The feedback law assumes that the transfer function of the resonator is symmetric, which is only an approximation of the BvD equivalent circuit of the piezoelectric resonator locally fitted by a parabolic law close to the resonance frequency. The resulting measurement is thus biased, but since the algorithm always operates at the same setpoint of equilibrating $y\left(f_{0} \pm f_{\text {step }}\right)$, this bias is constant as long as the quality factor of the resonator is not significantly changed by the interaction with the physical quantity under investigation.

Because a resonator is probed at known frequencies in a forced regime, the classical time-frequency uncertainty relationship will not apply: as opposed to a closed loop oscillator providing a frequency output to be monitored using a frequency counter, here the returned power response of a SAW resonator is probed at various known frequencies. Hence, the measurement bandwidth limitation is due to the loading and unloading duration of the resonator, not by a sampling duration since the (strong) assumption of a single mode resonator is considered, providing a single resonance frequency information, following a returned power dependence on frequency locally approximated by a parabolic function. Indeed, we will see that even though the measurement duration is less than 1 $\mathrm{ms}$, the resulting resonance frequency identification standard deviation is less than $1 \mathrm{kHz}$ (while a classical Fourier transform time-frequency uncertainty relationship would require the measurement duration to be inverse of the target frequency resolution).

\section{STRAIN GAUGE MEASUREMENT}

Having considered the maximum sampling rate of a SAW resonator using a two-point measurement strategy, the remaining issue concerns the maximum bandwidth of the observed phenomenon considering that each successive displacement of $f$ must be less than $f_{\text {step }}$. Assuming a (YXI)/40 quartz resonator, exhibiting a parallel stress sensitivity of $s_{\alpha 11}$ $=-16.7 \mathrm{ppm} / \mathrm{MPa}$ and $s_{\alpha 33}=+5.9 \mathrm{ppm} / \mathrm{MPa}$ transverse sensitivity, ${ }^{9}$ acting as a strain gauge with a linear relationship between stress $T$ and relative frequency shift $\left(f-f_{0}\right) / f_{0}$ through a coefficient ${ }^{10} s_{\alpha}: \frac{f-f_{0}}{f_{0}}=\Sigma s_{\alpha} T$, then during a time interval $t=1 / f_{s}$, with $f_{s}$ the sampling rate, the stress variation should induce a sensor resonance frequency variation of less than $f_{\text {step }}$ for the feedback loop to still operate. A periodic variation of the stress $T$ at angular frequency $\omega, T=T_{0} \exp (i \omega t)$, yields a condition $f_{0} s_{\alpha} T_{0}<f_{\text {step }} \frac{f_{s}}{\omega}$ or

$$
T_{0}<\frac{f_{\text {step }}}{f_{0}} \times \frac{f_{s}}{s_{\alpha} \omega} .
$$

This condition on the maximum stress variation in the strain gauge aims at keeping the feedback loop functional, depending on the physical property angular frequency. This case is applied here to the particular application of measuring the stress at the surface of a strain gauge bound to a musical tuning fork oscillating at about $440 \mathrm{~Hz}$ so that $\omega \simeq 2765 \mathrm{rad} \mathrm{s}^{-1}$. Since $s_{\alpha} \simeq 10^{-11} \mathrm{MPa} \mathrm{Hz}^{-1}$ for quartz, ${ }^{10,11}$ for a strain gauge operating at $434 \mathrm{MHz}$ and sampled at 10 $\mathrm{kHz}$, then $T_{0}<4.2 \mathrm{MPa}$. This limitation is significantly lower than the rupture stress of quartz of about $100 \mathrm{MPa} \cdot{ }^{12,13}$ For polychromatic vibrations, this consideration is extended by summing the contributions of each Fourier component of the measured signal and making sure the threshold condition is never met.

\section{WIRELESS STRESS MONITORING OF A VIBRATING STRUCTURE}

A demonstration experiment was carried out using a music tuning fork (440 Hz nominal resonant frequency) as vibrating structure. The tuning fork was instrumented at its base-where stress variation is maximum - with a $434 \mathrm{MHz}$ quartz SAW resonator acting as a stress gauge and interrogated through a wireless link (Fig. 2). The tuning fork was set in motion using a magnetically coupled voice coil, as 


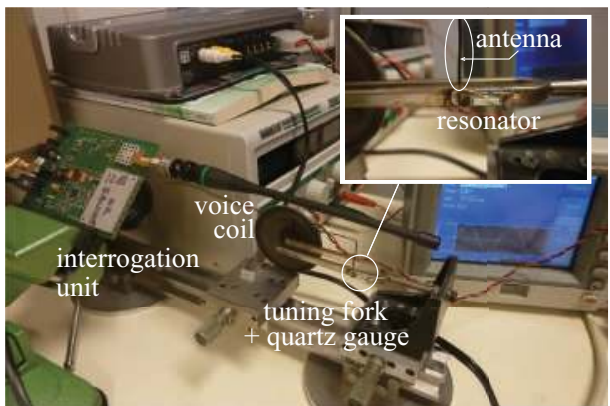

FIG. 2. Experimental setup: A music tuning fork is fitted with a quartz strain gauge resonator interrogated through a wireless link. The interrogation algorithm is implemented in the flexible digital interrogation unit.

described earlier. ${ }^{14}$ The two-point strategy was implemented as a fully digital algorithm in a flexible hardware interrogation unit fully controlled by a central processor (ARM7-core ADuC7026 microcontroller ${ }^{7}$ ), and the recorded resonance frequency was converted into an analog output voltage generated by the on-board $1 \mathrm{MS} / \mathrm{s}$ digital-to-analog converter (DAC) in order not to reduce the measurement bandwidth by the relatively slow asynchronous serial digital communication (Fig. 3). Indeed, even at the high baud rate of 57600 bauds, each symbol requires $174 \mu$ s for transfer through the digital link, i.e., more than the actual measurement duration using the above-presented two-point strategy.

The frequency-to-voltage conversion accounts for the direct digital synthesizer (DDS) word scaling: bits 12-23 of the

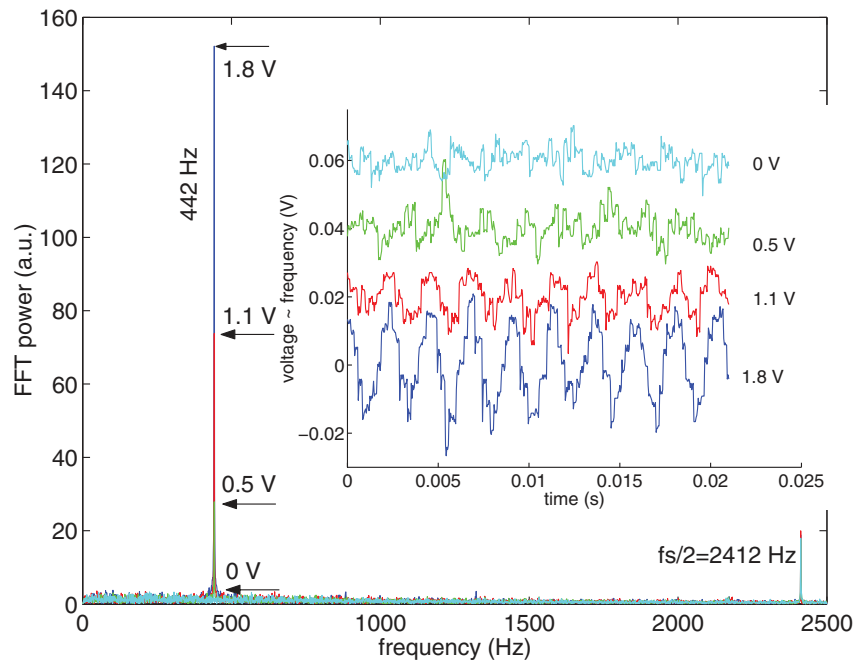

FIG. 3. Top right: Time domain records of the DAC output of the wireless acoustic sensor reader probing a quartz resonator strain gauge bound to a music tuning fork: the voice coil driving voltage at $442 \mathrm{~Hz}$ (maximum response amplitude) is increased from 0 to $1.8 \mathrm{~V}_{p p}$. As described in the text, the voltage-to-frequency conversion factor is $4.2 \times 10^{-6} \mathrm{~V} / \mathrm{Hz}$, so that the $20 \mathrm{mV}$ amplitude indicates a frequency shift amplitude of $362 \mathrm{~Hz}$ or a stress variation at the quartz strain gauge bound at the base of one of the prongs of about $650 \mathrm{kPa}$. Left: Fourier transform of the interrogation unit DAC voltage, sampled by a digital oscilloscope at $25 \mathrm{ksamples} / \mathrm{s}$. The strain gauge signal is visible at $442 \mathrm{~Hz}$ - the driving voltage at resonance of the tuning fork (set at $441.737 \mathrm{~Hz}$ ) - with a magnitude dependent on the driving signal amplitude, and vanishing when no excitation signal is applied $(0 \mathrm{~V})$. The interrogation unit sampling rate is visible at $f_{s} / 2=2412 \mathrm{~Hz}$, with a power independent on the voice coil driving voltage. 32-bit word defining the output frequency of an AD9954 DDS internally clocked at $200 \mathrm{MHz}$ are used to program a digitalto-analog voltage converted using a $3.3 \mathrm{~V}$ reference. Hence, the output voltage $V$ is related to the resonance frequency $f_{0}$ by

$$
f_{0} \times \frac{2^{32}}{200 \mathrm{MHz}} \times \frac{1}{4096} \times \frac{3.3 \mathrm{~V}}{4096}=4.2 \times 10^{-6} \mathrm{~V} / \mathrm{Hz}
$$

The observation of a $20 \mathrm{mV}_{p p}$ peak-to-peak output voltage as the tuning fork is vibrating close to its resonance frequency is thus associated to a resonance frequency variation the SAW resonator gauge of $4735 \mathrm{~Hz}_{p p}$. Using the tabulated ST-cut Rayleigh wave sensitivity ${ }^{10}$ of $-16.8 \mathrm{ppm} / \mathrm{MPa}$, as experimentally validated in Ref. 11, the stress variation at the base of a prong of the tuning fork is $650 \mathrm{kPa}_{p p}$.

The actual sampling rate of $f_{s}=4800 \mathrm{~Hz}$ (Fig. 3) is half the theoretical maximum update rate $(10 \mathrm{kHz})$ because of the additional mandatory digital processing steps: programming the 32-bit frequency defining register of the DDS through a $5 \mathrm{Mb} / \mathrm{s}$ SPI link already requires $6.25 \mu \mathrm{s}$, while further digital signal handling (data storage and arithmetic operations) on the $41.8 \mathrm{MHz}$ ARM7-core CPU yield an additional processing time of $24 \mu \mathrm{s}$.

Using the same apparatus and a stroboscopic imaging method, an end-prong displacement amplitude $d_{\max }$ of about 6 to $60 \mu \mathrm{m}$ has been observed, depending on the voice coil position. ${ }^{14}$ Assuming a distributed force acting on the whole length of the tuning fork prong considered as a clamped beam, the prong displacement equation along its length $x$ is

$$
d(x)=\frac{d_{\max }}{3 L^{4}} \times\left(4 L x^{3}-6 L^{2} x^{2}-x^{4}\right)
$$

with $d_{\max }$ the maximum displacement at position $L=8 \mathrm{~cm}$ (i.e., the length of the prong). Since the quartz strain gauge is located close to the clamping position of the prong at $x=0$, the bending stress at this position is

$$
\sigma=\frac{4 d_{\max }}{L^{2}} \times \frac{E h}{2},
$$

assuming a gauge of negligible thickness (quartz substrate thickness: $380 \mu \mathrm{m}$ ) with respect to the prong thickness $h=4$ $\mathrm{mm}$ (section $4 \times 5 \mathrm{~mm}^{2}$ ). Using an equivalent Young modulus of quartz $E=72 \mathrm{GPa},{ }^{15}$ the stress in the quartz strain gauge lies in the $\sigma=540-5400 \mathrm{kPa}$ range. The observed strain gauge frequency shift, associated with a stress of $650 \mathrm{kPa}$, lies within this interval.

While the two-point measurement strategy exhibits excellent measurement bandwidth, one drawback is the reduced interrogation range: since the resonator is probed on purpose at frequencies different from the resonant frequency, the loading efficiency is poor due to the lack of synchronism between the incoming electromagnetic wave and the SAW interdigitated transducer and Bragg grating geometry. A $10 \mathrm{~dB}$ loss (20 dB two-way trip) in free space propagation loss (FSPL) has been observed with respect to a strategy aimed at probing a resonator close to its resonance frequency.

One option to yet increase the measurement speed consists in reducing the quality factor of the resonator. Using a resonator with such a quality factor allows for shortening the interrogation pulse duration but yields reduced accuracy 

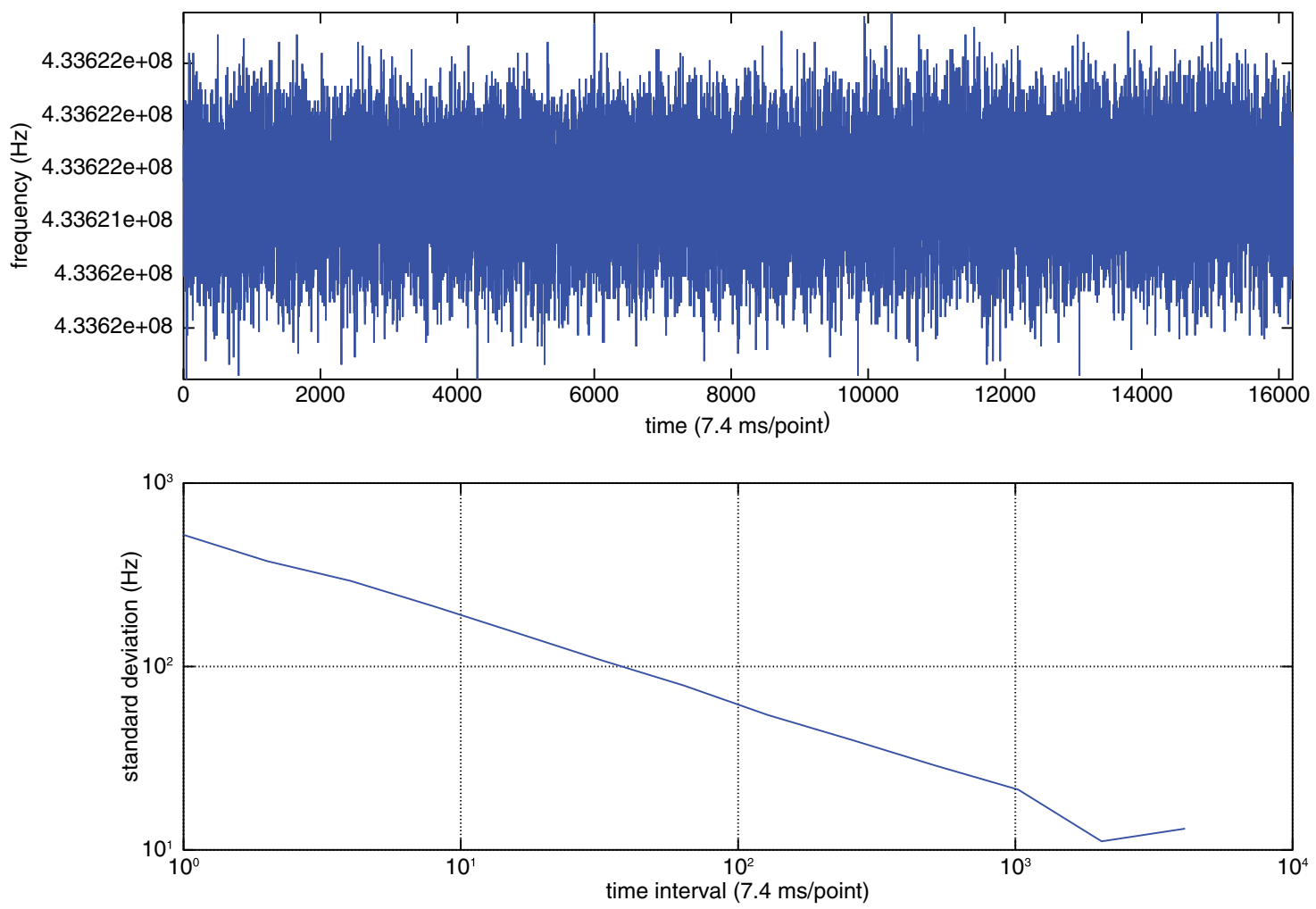

FIG. 4. Top: Two-minute measurement of the frequency output of the resonator interrogation unit running the two-point algorithm, here with a digital communication of the measurement through an asynchronous serial link, yielding a rather slow measurement rate of $135 \mathrm{~Hz}$. Bottom: Allan deviation of the resulting dataset, exhibiting a sub-kHz standard deviation (two-point standard deviaton) and sub-100 Hz Allan deviation at $1 \mathrm{~s}$ (135 sample averages) integration time.

and interrogation distance. The same result is obtained by shortening the interrogation pulse width while probing a high quality factor resonator. In the latter case, because probing an acoustic resonator with a wideband pulse physically corresponds to a convolution of the acoustic and probe pulse time domain responses (or product in the frequency domain), the high quality factor of the sensor is no longer usable because the pulse spectral shape no longer matches the sensor spectral occupation (most of the incoming energy lies outside the bandpass of the resonator). Hence, high quality factor resonators are hardly compatible with measurements above the $50 \mathrm{kHz}$ range. The current implementation, using a long probe pulse (spectral width narrower than the width at half height of the acoustic resonance), exhibits a sub-kHz standard deviation on the resonance frequency identification, decreasing to sub$100 \mathrm{~Hz}$ when running a 100 point sliding average, as shown by the Allan deviation plot of Fig. 4.

A quantitative design rule of a resonator targeted for high bandwidth measurement, aimed at estimating the interrogation distance loss tradeoff associated with increased measurement speed allowed by lowering the quality factor from an initial value of $Q_{1}$ to an improved value $Q_{2}$ (through a novel sensor design), is proposed as follows:

1. The radiofrequency link budget is characterized by the antenna efficiency, FSPL, losses induced in the sensor, and emitted to received power ratio. For a given interrogation unit equipped with a given set of antennas, only the losses in the resonator and the FSPL are expected to vary.
2. On the one hand the loading delay of the resonator is $Q /\left(\pi f_{0}\right)$ which is representative of the duration needed to perform a measurement at best conditions and on the other hand the FSPL in a RADAR system is a function of $1 / d^{4}$ where $d$ is the distance between the interrogation unit and the sensor.

3. Assuming the interrogation unit samples at a fixed delay $t_{m}$ the signal returned from the resonator after loading at frequency $f$, then the exponentially decaying return signal envelope is given by $\exp \left(-t_{m} \times \pi f_{0} / Q\right)$, or, using a logarithmic scale, the difference between the return signal losses due to the exponentially decaying envelope between resonators of quality factor $Q_{1}$ and $Q_{2}$ respectively is as follows:

$$
8.7 \times \pi f_{0} t_{s}\left(\frac{1}{Q_{1}}-\frac{1}{Q_{2}}\right),
$$

where $20 \times \log _{10}(e) \simeq 8.7$.

4. Because the FSPL depends as the fourth power of the interrogation distance, the variation of the interrogation distances $d_{1}$ and $d_{2}$ when probing the two resonators with quality factors $Q_{1}$ and $Q_{2}$ respectively is given by

$$
\begin{gathered}
40 \log _{10}\left(\frac{d_{2}}{d_{1}}\right)=8.7 \times \pi f_{0} t_{s}\left(\frac{1}{Q_{1}}-\frac{1}{Q_{2}}\right) \\
\Leftrightarrow \frac{d_{2}}{d_{1}}=10^{-\frac{8.7}{40} \times \pi f_{0} t_{s}\left(\frac{1}{Q_{2}}-\frac{1}{Q_{1}}\right) .}
\end{gathered}
$$


As a numerical application, if $f_{0}=434 \mathrm{MHz}, t_{s}=1 \mu \mathrm{s}$ allowing for the electromagnetic clutter to fade out and the RF duplexer to stabilize, $Q_{1}=10000$ and $Q_{2}=5000$, then the interrogation distance is only reduced by $7 \%$ (since $d_{2} / d_{1}$ $=0.934$ ) while the interrogation bandwidth is doubled. Hence, depending on the targeted application, if interrogation range is not a mandatory requirement, decreasing the quality factor following the described rules will significantly increase the measurement bandwidth while inducing marginal interrogation distance loss. Since probing a high quality factor resonator with a short pulse whose spectral width is wider than the resonator width at half height, the equivalent sensor quality factor is lowered and the same result is achieved with no need to actually change the sensor hardware. This rule is also applicable when comparing the interrogation range of piezoelectric resonators and dielectric resonators which might act as passive radiofrequency $\operatorname{sensors}^{16}(Q \sim 100$ at $434 \mathrm{MHz}$ (Ref. 17)): in the latter case, the interrogation distance is divided by 1000 . An additional drawback of reducing the quality factor, beyond the loss of interrogation range, is the decreased measurement resolution due to the broader resonance.

\section{CONCLUSION}

Using a digital implementation on a flexible monostatic radiofrequency pulsed RADAR hardware requiring only two sampling points, a feedback controlled strategy for probing the resonant frequency of a passive resonator acting as a sensor monitored through a wireless link has been demonstrated. This approach has been implemented to quantify the corresponding system bandwidth, as this fast algorithm interrogation approach is discussed to allow for real time data acquisition, for instance of dynamic strain measurements with a refresh rate better than $200 \mu \mathrm{s}$. A $5 \mathrm{kHz}$ sampling rate has been experimentally emphasized, which is better than any system based on Fourier Transform analysis considering the accuracy of the proposed system. ${ }^{18}$ There is still possibility to theo- retically increase this sampling rate to $10 \mathrm{kHz}$, either by improving the signal processing implementation or adapting the sensor characteristics, yielding tradeoff between refresh rate, accuracy and interrogation distance.

${ }^{1}$ L. Reindl, G. Scholl, T. Ostertag, H. Scherr, U. Wolff, and F. Schmidt, IEEE Trans. Ultrason. Ferroelectr. Freq. Control 45, 1281 (1998).

${ }^{2}$ V. Plessky and L. Reindl, IEEE Trans. Ultrason. Ferroelectr. Freq. Control 57, 654 (2010).

${ }^{3} \mathrm{C}$. Hartmann and L. Claiborne, in 2007 IEEE International Conference on RFID, Grapevine, Texas, USA, 2007.

${ }^{4}$ L. Reindl, A. Pohl, G. Scholl, and R. Weigel, IEEE Sens. J. 1, 69 (2001).

${ }^{5}$ C. Droit, G. Martin, S. Ballandras, and J.-M. Friedt, Rev. Sci Instrum. 81, 056103 (2010).

${ }^{6} \mathrm{~J}$. Beckley, V. Kalinin, M. Lee, and K. Voliansky, in IEEE International Frequency Control Symposium and PDA Exhibition, New Orleans, Louisiana, USA (IEEE, 2002), pp. 202-212.

${ }^{7}$ J.-M. Friedt, C. Droit, G. Martin, and S. Ballandras, Rev. Sci. Instrum. 81, 014701 (2010).

${ }^{8}$ See supplementary material at http://dx.doi.org/10.1063/1.4705728 for a typical resonator transfer function measurement and the associated parameters extracted from a least square fit which are used in numerical applications throughout this document.

${ }^{9}$ S. Ballandras and E. Bigler, J. Appl. Phys. 72, 3272 (1992).

${ }^{10}$ E. Bigler and S. Ballandras, in Proceedings of the 1996 IEEE International Frequency Control Symposium, Honolulu, Hawaii, USA (IEEE, 2009), pp. 422-429.

${ }^{11}$ G. Bruckner, G. Stampf, G. Franz, R. Fachberger, and R. Hauser, in Proceedings of the 2009 IEEE International Ultrasonics Symposium, Roma, Italy (IEEE, 2009), pp. 823-826.

${ }^{12}$ C. Scholz, J. Geophys. Res. 77, 2104, doi:10.1029/JB077i011p02104 (1972).

${ }^{13} \mathrm{R}$. Ward, in 14th Piezoelectric Device Conference and Exhibition, Kansas City, USA (IEEE, 1992), Vol. 2, pp. 61-70.

${ }^{14}$ P. Sandoz, J.-M. Friedt, E. Carry, B. Trolard, and J. G. Reyes, Am. J. Phys. 77, 20 (2009).

${ }^{15}$ X. Chen, S. Zhang, G. Wagner, W. Ding, and R. Ruoff, J. Appl. Phys. 95, 4823 (2004).

${ }^{16}$ H. Hallil, P. Menini, and H. Aubert, in 2009 IEEE Sensors, Christchurch, New Zealand (IEEE, 2009), pp. 226-228.

${ }^{17}$ D. Hanson, "Microwave resonators," in Dielectric Resonators, 3rd ed., edited by D. Kajfez and P. Guillon (Noble Publishing Corporation, Atlanta, GA, 1998).

${ }^{18}$ V. Kalinin, R. Lohr, A. Leigh, J. Beckley, and G. Bown, in EFTF - 24th European Frequency and Time Forum, Noordwijk, The Netherlands, 2010. 